\title{
Psychosozialer Notfall \\ Substanzinduzierte Störungen durch illegale Drogen - Teil 1
}

Michael Kinn • Rüdiger Holzbach • Frank-Gerald Bernhard Pajonk

Notfälle wegen Drogenkonsums sind oft schwer zu beherrschen. Zum einen fehlen diagnostische Möglichkeiten vor Ort, zum anderen kann der Notarzt nur symptomatisch behandeln. Selten sind Drogenintoxikationen auf den Konsum von nur einer Substanz zurückzuführen - und oft stehen suizidale Absichten hinter der Intoxikation. Das schwierige Umfeld, in dem es zu Drogennotfällen kommt, erschwert das Handeln zusätzlich. Einsätze im Zusammenhang mit einer Entzugssymptomatik eröffnen die Möglichkeit, Betroffene in eine therapiegestützte Abstinenz zu überführen - diese Chance sollte nicht vertan werden.

Abb. 1, links Haschisch in Platten.

Abb. 2, Mitte Kugelähnlich geformte Stücke von Cannabisharz. Es ist die Vorstufe von Haschisch (darüber im Bild).

Abb. 3, rechts Eine noch ungetrocknete Hanfpflanze.
Lage in Deutschland Der Drogen- und Suchtbericht der Bundesregierung für das Jahr 2007 zeichnet ein gemischtes Bild zum Drogenkonsum in Deutschland:

- Die Zahl der Erstkonsumenten von Cannabis war erstmals rückläufig.

- Die Zahl der „starken Kiffer“ ist mit rund 600000 annähernd gleich geblieben.

- Unter den erstauffälligen Konsumenten harter Drogen ging die Anzahl der Konsumenten von Heroin, Kokain und Ecstasy zurück.

- Hingegen stieg die Zahl der erstauffälligen Konsumenten von (Meth-)Amphetamin, Crack und sonstigen Drogen (insb. Halluzinogene) [1].

Insgesamt dürfte die tatsächliche Zahl der Drogenkonsumenten aber um den Faktor 7 höher liegen [2].

\begin{abstract}
Akute Intoxikationen mit Opiaten und Opioiden, Suizide, Unfälle unter Drogen und Infektionskrankheiten wie HIV oder Hepatitis B und C fordern jedes Jahr Menschenleben. Erstmals seit dem Jahr 2000 stieg 2007 wieder die Zahl der Drogentoten in Deutschland: auf 1394 Tote (+ $8 \%$ zum Vorjahr) [1].
\end{abstract}

Stadt-Land-Gefälle Drogen und Suchtmittel sind immer wieder Grund für Notarzt-Einsätze. In deutschen Großstädten liegt hier die Rate bei $11 \%$ aller psychisch auffälligen Patienten, in ländlichen Regionen ist die Rate deutlich niedriger (3\%) [3]. Ursächlich für Notfallsituationen im Zusammenhang mit Suchtmitteln können sein: Intoxikationen, Entzüge, Folgeschäden des protrahierten Konsums, allergische Reaktionen durch Begleit- und Streckmittel sowie akzidentiell Situationen, die Folge der Bewusstseinseinschränkung sind (inkl. Trauma, Unfälle, psychischer Erregungszustand).

- Der Konsum von Suchtmitteln ist oft mit Suizidalität vergesellschaftet.

- Alle Notfälle mit Suchtmitteln bergen die Gefahr, wichtige Differenzialdiagnosen zu übersehen.
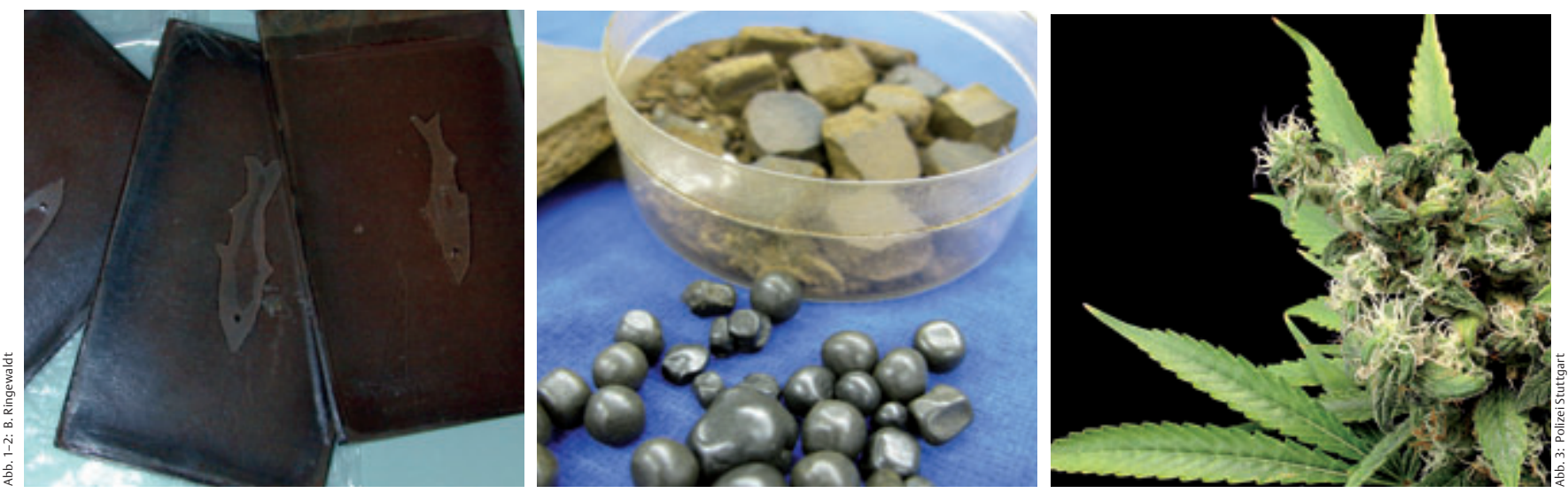
Wirkung und Konsumgründe Suchtmittel wirken interindividuell unterschiedlich. Abhängig von Körpermasse, Geschlecht, momentaner Stimmung, Begleitsubstanzen, Vorerkrankungen, Gewöhnung und Umfeld können gleiche Mengen eines Suchtmittels unterschiedliche Rauschzustände hervorrufen. Die akzidentielle Überdosierung ist häufig und mitunter lebensbedrohlich [4]. Der Konsum von Suchtmitteln hat verschiedene Ursachen. Süchtiges Verhalten rührt oftmals daher, dass mit Hilfe eines Suchtmittels

- persönliche Stimmungen verbessert,

- Sorgen und Probleme verdrängt und

- vermeintliche Auswege aus unangenehmen Situationen geschaffen werden.

Auch eine subjektive Leistungssteigerung kann Grund für den Konsum sein.

Der Suchtbegriff Von einer Sucht spricht man, wenn ...

- keine Kontrolle mehr über Beginn, Beendigung und Menge der Einnahme eines Suchtmittels besteht.

- der Wunsch oder sogar der Zwang besteht, immer wieder eine bestimmte Substanz zu konsumieren.

- persönliche Interessen zugunsten der Suchtmitteleinnahme oder Beschaffung vernachlässigt werden.

- eine Toleranzwirkung einsetzt, körperliche Entzugssymptome auftreten oder der Konsum der Substanz trotz schädlicher Folgen fortgesetzt wird.

Drogenassoziierte Notfälle sind häufig, dem Suchtmittelkonsum liegt süchtiges Verhalten zugrunde. Dabei werden Notärzte häufiger mit Intoxikationen als mit Entzugssyndromen konfrontiert.

Der Entzug von einem Suchtmittel ist selten lebensbedrohlich - mitunter aber sehr unangenehm für die Betroffenen. Daher kann man mit einer Vor-Ort-Motivation zum Entzug und mit dem Angebot adäquater Hilfe eine anhaltende Abstinenz erreichen.

\section{Illegale Drogen}

\section{Cannabis}

Konsumvarianten Cannabis kann inhalativ oder oral konsumiert werden. Man unterscheidet verschiedene Zubereitungsformen, z.B. Haschisch (aus Cannabisharz; $\bigcirc$ Abb. 1, 2), Marihuana (Cannabiskraut; $\bullet$ Abb. 3) oder Haschischöl (Cannabisharzextrakt).

- Bei inhalativem Konsum mittels Taschenrauchgeräten und Joints ( $\bullet$ Abb. 4 ) oder Was- serpfeife tritt die Wirkung binnen 30 s ein und kann bis zu $3 \mathrm{~h}$ andauern.

- Der orale Konsum in fetthaltigen Getränken (Kakao) oder Backwaren ist durch eine langsamere Resorption (30-60 min) und eine bis ('weiter auf $\bigcirc$ S. 749)

Tab. 1 nach [9]

\section{Symptome des protahierten Kokainkonsums}

\begin{tabular}{|c|c|}
\hline neurologisch & $\begin{array}{l}\text { Koordinationsstörungen } \\
\text { Verwirrtheit } \\
\text { - Unruhe und zerebrale Krampfanfälle } \\
\text { Dyskinesien } \\
\text { Dystonien } \\
\text { Hyperpyrexien mit Koma } \\
\text { zerebrale Minderdurchblutung durch Vaso- } \\
\text { konstriktion mit ischämischen Läsionen sowie } \\
\text { Parenchym- und Subarachnoidalblutungen } \\
\text { weiterhin neurokognitive Störungen von } \\
\text { Aufmerksamkeit, Gedächtnis- und Lernfähigkeit } \\
\text { sowie Verlust von verbalen und visuomotorische } \\
\text { Fähigkeiten }\end{array}$ \\
\hline kardiovaskulär-pulmonal & $\begin{aligned} \text { - kardiale Arrythmien } \\
\text { Atemdepressionen } \\
\text { - Brustschmerzen } \\
\text { - Husten } \\
\text { - Bronchitiden } \\
\text { - Hämoptysen } \\
\text { - Pneumonien } \\
\text { alveoläre Blutungen } \\
\text { „Crack-Lunge“ (bei Inhalation treten neben } \\
\text { Brustschmerzen, Dyspnoe und Fieber klinische } \\
\text { Symptome einer Pneumonie ohne radiologische } \\
\text { Zeichen auf) } \\
\text { Pneumothorax und spontanes Pneumo- } \\
\text { mediastinum }\end{aligned}$ \\
\hline $\begin{array}{l}\text { allgemeine körperliche } \\
\text { Symptome }\end{array}$ & $\begin{array}{l}\text { Niereninsuffizienz mit Rhabdomyolyse } \\
\text { Nasenblutungen } \\
\text { Sinusitiden } \\
\text { - korneale Defekte } \\
\text { - Nasopharyngealnekrosen } \\
\text { gastrointestinale Ischämien mit gastro- } \\
\text { duodenalen Ulcera } \\
\text { - Priapismus und Penisnekrosen }\end{array}$ \\
\hline Sexualität & $\begin{array}{l}\text { Erhöhung von Libido und sexueller Erregbarkeit } \\
\text { auf Grund dopaminerger Wirkung }\end{array}$ \\
\hline Schwangerschaft & $\begin{array}{l}\text { sog. „Jittery-Baby-Syndrome“: } \\
\text { retardiertes Wachstum des Fötus, erniedrigtes } \\
\text { Geburtsgewicht, verminderte Plazentadurch- } \\
\text { blutung und fetale Hypoxie, vorzeitige Plazenta- } \\
\text { ablösung und Spontanaborte }\end{array}$ \\
\hline $\begin{array}{l}\text { Störungen bei Kindern } \\
\text { Kokainabhängiger }\end{array}$ & $\begin{array}{l}\text { niedriges Geburtsgewicht } \\
\text { reduziertes Größenwachstum } \\
\text { verminderter Kopfumfang } \\
\text { Spastik } \\
\text { erhöhter Extensorentonus } \\
\text { Hypereflexie und Krampfneigung } \\
\text { Bei mütterlichem Drogenkonsum ist Kokain } \\
60 \text { h in der Muttermilch nachweisbar und } \\
\text { kann Apnoeanfälle beim Neugeborenen hervor- } \\
\text { rufen. }\end{array}$ \\
\hline
\end{tabular}




\section{Fallbeispiel}

\section{Der Fall}

- Als Einsatzgrund nennt die Leitstelle einen „kardialen Notfall“. In einem Vorstadt-Reihenhaus öffnete ein 22-jähriger Mann dem Notarzt. Er berichtete, dass er vor 4 Tagen Gras geraucht habe, das er von einem anderen Lieferanten als dem üblichen erhalten habe. Ihm sei danach ganz merkwürdig geworden, sein Herz habe begonnen, schneller zu schlagen, er habe plötzlich Angst bekommen. Außerdem habe sich die Welt in ganz merkwürdiger Weise um ihn verzerrt. Alles sei unwirklich geworden. Er habe gedacht, dass dies vielleicht an der Qualität des Marihuanas gelegen habe. Die Beschwerden seien nach 2 Tagen abgeklungen.

Heute habe er nun wieder Gras von seinem bekannten Lieferanten geraucht. Die Symptome seien sofort wieder aufgetreten, diesmal nur noch schlimmer. Er habe das Gefühl, sein Herz habe sich im Brustkorb vergrößert, verdränge die Lunge und schlage sehr heftig und wie dröhnend an seinen Brustkorb. Er bekomme daher keine Luft mehr zum Atmen. Außerdem wirkten ihm gut bekannte Menschen plötzlich bedrohlich, sie sähen ihn auf eine gewisse Art an, als würden sie etwas von ihm erwarten. Er habe eine Riesenangst, dass er da nicht mehr herauskomme.

Anamnese, Diagnostik, Therapie Auf nähere Nachfrage berichtet er, dass er seit 6 Jahren regelmäßig Marihuana konsumiere, seit etwa 1 Jahr ca. 1-2 Gramm pro Tag, vorher weniger, sowohl als „Tüte“ als auch als „Bong“. Solche Symptome seien vorher nie aufgetreten. Andere Drogen nehme er nicht.

Der Blutdruck beträgt 120/70 mmHg, Puls: 72/min, Blutzucker: $86 \mathrm{mg} / \mathrm{dl}, \mathrm{SO}_{2}$ $99 \%$. Das EKG zeigte einen Sinusrhythmus, Rhythmus, Erregungsleitung und -rückbildung sind nicht gestört.

Der Patient erhält initial $1 \mathrm{mg}$ Lorazepam oral. Bereits auf dem Weg in die Notaufnahme berichtet er über eine deutliche Reduktion seiner ängstlichen Befürchtungen. Die wahnhaft anmutenden körperlichen und psychischen Symptome verschwinden ebenfalls sukzessive.

Weiterer Verlauf Die Ergebnisse der weiteren körperlichen und apparativen Untersuchungsverfahren waren unauffällig. Im toxikologischen Screening wurde lediglich Cannabis nachgewiesen. Es erfolgte ein psychiatrisches Konsil. Der Patient wurde dringend auf die Notwendigkeit einer Abstinenz bei V.a. das Vorliegen einer drogeninduzierten Psychose hingewiesen. Anschließend wurde er auf eigenes Drängen nach Hause entlassen.

\section{Symptome des Kokainentzugssyndroms}

\begin{tabular}{|l|l|l|}
\hline Phase & Dauer & Symptome \\
\hline 1. „crash“ & 1-2 Tage & $\begin{array}{l}\text { starke Dysphorie, Anhedonie, } \\
\text { Schlaflosigkeit, Irritabilität, Angst, } \\
\text { Kokaincraving } \\
\text { später Lethargie und Anergie }\end{array}$ \\
\hline 2. „withdrawal“ & $1-10$ Wochen & $\begin{array}{l}\text { > mit Hypersomnolenz von ca. 8-50 h } \\
\text { dann 1-4 Tage euthyme Stimmungs- } \\
\text { lage mit normalem Schlafmuster, } \\
\text { im Anschluss erneut Anhedonie, } \\
\text { milde Dysphorie, Angst, Irritabilität } \\
\text { und Anergie }\end{array}$ \\
\hline 3. „extinction“ & Monate bis Jahre & $\begin{array}{l}\text { "craving“: starkes Verlangen } \\
\text { nach der Droge }\end{array}$ \\
\hline
\end{tabular}

Tab. 2 nach [11] 


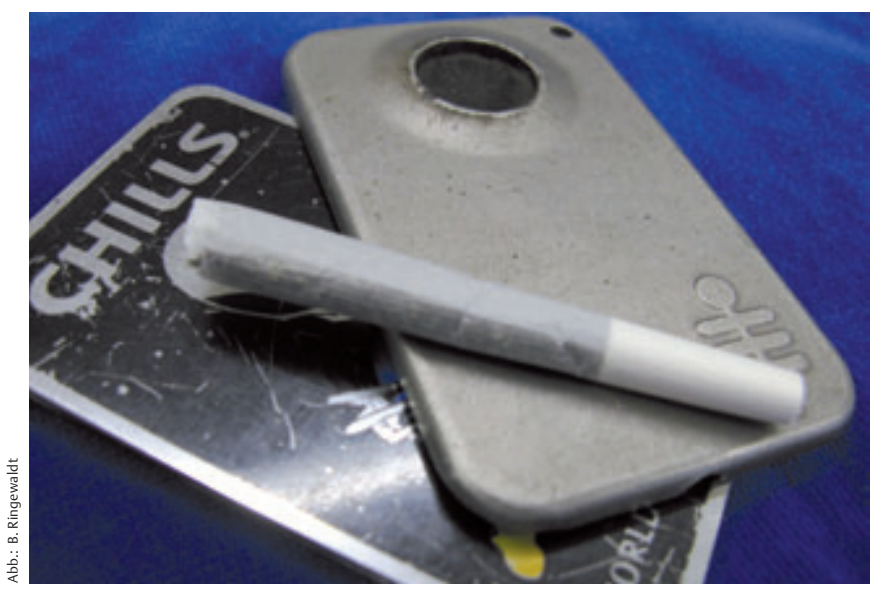

zu $6 \mathrm{~h}$ andauernde, wellenartig modulierte Wirkdauer gekennzeichnet. Die orale Einnahme lässt sich nur sehr schwer steuern und ist häufig Grund für eine Überdosierung.

Cannabis enthält Delta-9-Tetrahydrocannabinol (THC) $[5,6]$ und wirkt über die G-Proteingekoppelten Membranrezeptoren $\mathrm{CB}_{1}$ und $\mathrm{CB}_{2}$, die sich in hoher Anzahl im Hippokampus, in den Basalganglien, im Kleinhirn und in der Amygdala befinden [7].

Intoxikation Körperliche Symptome einer Cannabisintoxikation sind: Tachykardie, Übelkeit, Erbrechen, Schwindel, gestörtes Temperaturempfinden, gerötete Augen, erweiterte $\mathrm{Pu}$ pillen, trockener Mund. Psychische Symptome bestehen typischerweise in Erregung, Angst, Aggression und psychotischem Erleben. Es können ein verändertes Raum-Zeit-Empfinden oder eine veränderte Urteils- und Kritikfähigkeit auftreten. Reine Cannabisintoxikationen sind nicht lebensbedrohlich, die Symptome klingen entsprechend der Halbwertszeit nach wenigen Stunden ab. Der protrahierte Konsum kann aber zu bleibenden Psychosen führen. Differenzialdiagnostisch müssen in Betracht gezogen werden:
- Mischintoxikationen

- Intoxikationen durch andere psychotrope Substanzen

- schizophrene Psychosen

Versorgung bei Cannabis-Intoxikation Die Behandlung sollte symptomatisch erfolgen.

- Auf Betroffene sollte beruhigend eingegangen werden, bei starker Erregung kann man Benzodiazepine (z.B. Diazepam 5-10 mg i.v.) geben.

- Stehen psychotische Symptome im Vordergrund, so sollte besser ein Antipsychotikum (z.B. Haloperidol 5-10mg i.v.) verabreicht werden.

- Bereits bei vermuteter Eigen- oder Fremdgefährdung sollte die Unterbringung nach Unterbringungsgesetz erfolgen.

Entzug Patienten, die regelmäßig Cannabis konsumieren, leiden häufig unter Depressionen mit einer Antriebsminderung, Lethargie und Anhedonie (sog. amotivationelles Syndrom) sowie Ängsten. Zudem können sogenannte „flashbacks“ (veränderte Wahrnehmung ohne erneuten Cannabiskonsum), psychotische Symptome und Persönlichkeitsveränderungen auftreten [8]. Entzugserscheinungen nach Cannabiskonsum können Reizbarkeit, Ruhelosigkeit, Schlafstörun-
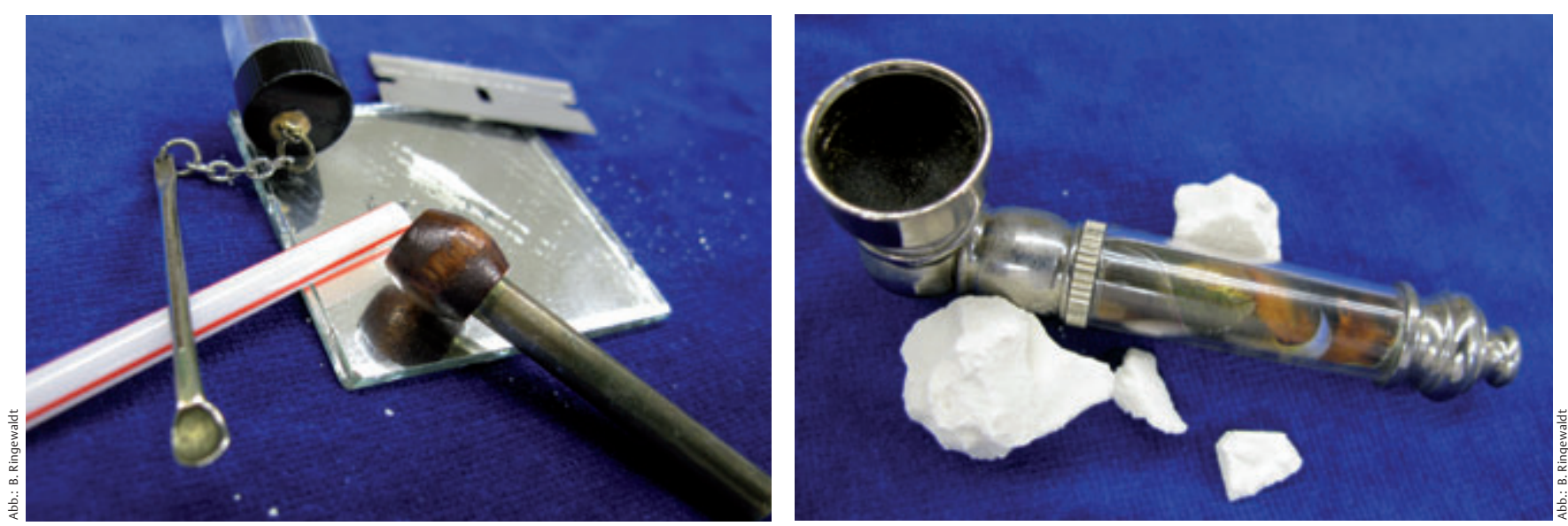

Abb. 4, links Die kreditkartengroßen Taschenrauchgeräte liegen momentan unter HaschischKonsumenten im Trend: Man raucht die Substanz pur ohne Tabak. Anders der herkömmliche Joint, bei dem das Haschisch nach Erhitzen in den Tabak gebröselt wird.

Abb. 5, rechts Oben (weiß): Kokain vom Stein; darunter Kokain aus Bodypacks, hier in sehr hoher Qualität. Rechts ein Portionierer aus Glas für Kokainpulver.

O Abb. 6, links Oben ein Glasgefäß für Kokainpulver mit Schraubverschluss, an dem ein Portionierungslöffelchen hängt. Auf der Spiegelfläche eine „Line“. Darunter, zum Inhalieren: ein Strohhalm und ein Röhrchen mit Holznuss, die das Nasenloch beim Inhalieren abdichtet.

- Abb. 7, rechts Crackpfeife mit Crackbrocken. Etwa die Hälfte des untersten Brockens (rechts) genügt für einen erheblichen Rauschzustand. 
Abb. 8, links Roh-Opium, wie man es braucht, um daraus Heroin oder Morphine herzustellen.

Abb. 9, rechts Meistens bräunlich, seltener weiß: Heroin wird in Pulverform gehandelt und riecht nach Essig.
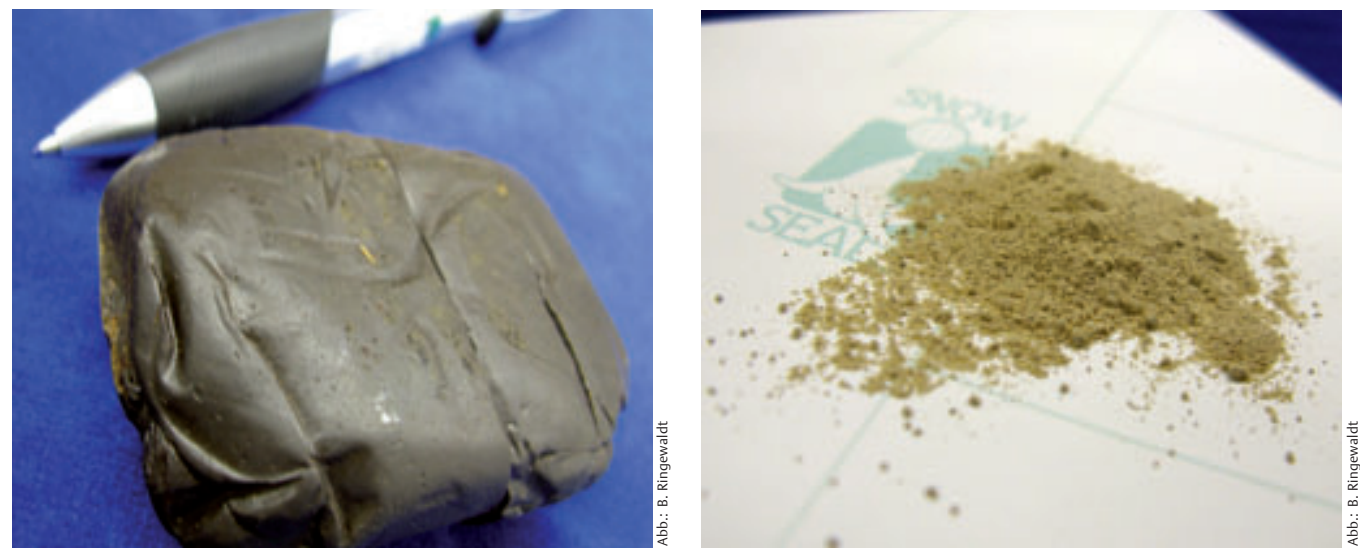

gen, vermehrter REM-Schlaf, Appetitlosigkeit, Frösteln und Zittern sein. Es besteht keine vitale Bedrohung.

Cannabis zählt zu den weichen Drogen. Weder Intoxikation noch Entzug stellen eine vitale Bedrohung dar. Wichtig bei psychotischen Symptomen ist die differenzialdiagnostische Abklärung von Psychosen aus dem schizophrenen Formenkreis.

\section{Kokain}

Wirkungsweise Kokain ( Abb. 5) bewirkt eine verstärkte Freisetzung von Dopamin und hemmt die Wiederaufnahme von Dopamin, Serotonin und Noradrenalin aus dem synaptischen Spalt im ZNS [8]. Es wird aus den Blättern des Kokastrauches gewonnen und kann je nach Zubereitungsform geraucht, gespritzt, enteral oder über Schleimhäute (z.B. geschnupft; - Abb. 6) aufgenommen werden. Der schnellste Wirkeintritt wird beim Rauchen der Kokainbase (Freebase) oder des Kokainhydrochlorids (Crack; - Abb. 7) nach wenigen Sekunden erreicht. Enteral zugeführt dauert die Resorption ca. $60 \mathrm{~min}$. Die Plasmahalbwertszeit beträgt 40-60 min.

- Kokain wirkt enthemmend, steigert das Selbstbewusstsein und die subjektive Leistungsfähigkeit.

Tab. 3 nach [9]
Intoxikation Die akute Kokainintoxikation ist mitunter lebensbedrohlich. Überdosierung oder protahierter Konsum können eine Vielzahl an somatischen Symptomen hervorrufen ( $\bullet$ Tab. 1). Vital bedrohlich sind:

- Hypertonie bis zur hypertensiven Krise mit Hirnmassenblutung

- Tachykardie

- pektanginöse Beschwerden bis zum Herzinfarkt

- Rhythmusstörungen

- Krampfanfälle bis zum Status epilepticus Therapeutisch kaum zu beherrschen sind die maligne Hyperthermie und der Atemstillstand über eine Tachypnoe.

\section{Psychische Folgen einer Kokainintoxikation} können Panikstörungen, ängstlich-wahnhaftes Erleben, Dermatozoenwahn und andere Halluzinationen sowie Suizidversuche bzw. Suizide sein [8-10].

Versorgung bei Intoxikation Neben der Erhebung und Sicherung der Vitalfunktionen beschränkt sich die Therapie der Kokainintoxikation auf die akute Symptomatik.

- Bei einer Hypertonie kann man eine Therapie mit $\alpha$-Blockern (z.B. Urapidil), $\alpha 2$-Agonisten (z.B. Clonidin $0,15 \mathrm{mg}$, langsam i.v.) oder in schwereren Fällen mit Natrium-Nitroprussid (2-10 ugg/kg KG/min) oder mit Glycerol-Trinitrat (2-8 mg/h) im Perfusor versuchen [10].

- Die Applikation von $\beta$-Blockern sollte mit Bedacht und nur in Kombination mit Vasodilatoren oder $\alpha$-Blockern geschehen.

- Lidocain eignet sich zur Therapie ventrikulärer Tachyarrhythmien, senkt aber die ohnehin erniedrigte Krampfschwelle und sollte deshalb mit Benzodiazepinen kombiniert werden.

- Benzodiazepine sollten ebenfalls bei akuter Erregung zum Einsatz kommen (z.B. Diazepam 5-10 mg i.v.).

- Bei psychotischem Erleben ist die Kombination mit z.B. Haloperidol sinnvoll. 


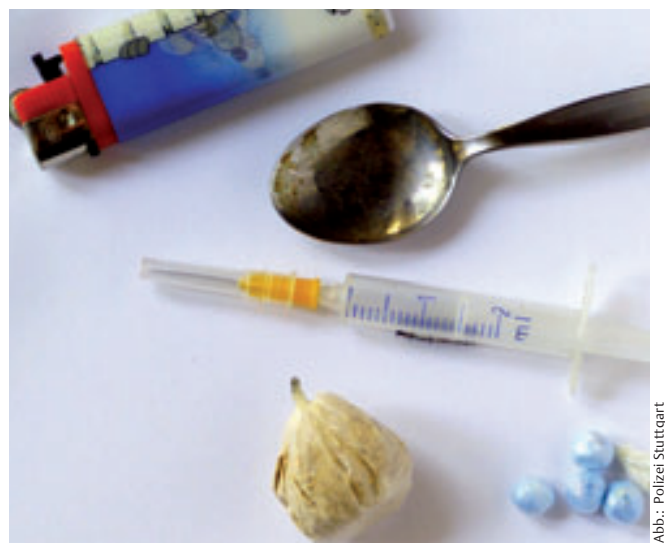

Das toxische Lungenödem sollte mittels Diuretika, PEEP-Beatmung und Kortikosteroiden behandelt werden.

Entzug Ein Kokainkonsum verursacht schwerste psychische Abhängigkeit. Die sich dem Rausch anschließenden depressiven Phasen sind verursacht durch einen relativen Mangel an Neurotransmittern nach Entleerung präsynaptischer Zellen und herunterregulierter Rezeptordichte.

- Der Entzug ist physisch unproblematisch, die psychischen Symptome können jedoch bis zur Suizidalität reichen ( $\bullet$ Tab. 2).

Versorgung im Entzug Patienten im Kokainentzug sollten verbal beruhigt werden (Talking down). Die weitere Behandlung erfolgt symptomatisch:

- Dysphorie, Unruhe und Angst: Therapie mit Benzodiazepinen (z.B. 5-20 mg Diazepam i.v.)

- psychotische Symptome: mit Antipsychotika (z.B. Haloperidol 5-10 mg i.v.)

- Hypertonie und Tachykardie können bei gesicherter Drogenabstinenz mittels $\beta$-Blockern (z.B. Propanolol $5 \mathrm{mg}$ i.v.) oder besser $\alpha 2$-Agonisten (z.B. Clonidin 0,15 mg i.v.) behandelt werden.

- Die Einweisung in eine psychiatrische Klinik zur Abstinenzstützung und Differenzialdiagnose bei psychotischen Störungen ist sinnvoll.

Kokain birgt ein immenses psychisches Abhängigkeitspotenzial. Die Intoxikation ist mitunter lebensbedrohlich und kann auch beim jungen Patienten Infarkte und Insulte auslösen.

\section{Opiate und Opioide \\ $\nabla$}

Wirkeigenschaften Opiate sind Alkaloide des Opiums ( Abb. 8) und begleiten die Menschheit

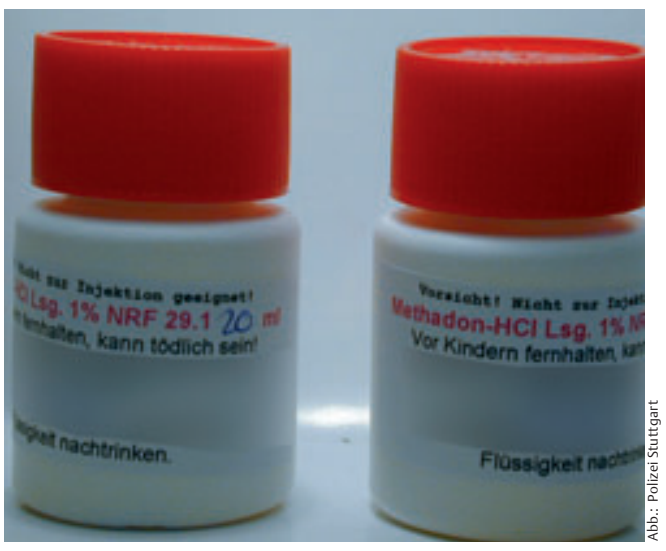

Abb. 10, links Fixerbesteck: Unter dem Feuerzeug ein Löffel zum Aufkochen und eine Spritze. Darunter in der Mitte wasserdicht verpacktes Drogenmaterial. Unten rechts: Heroin, verpackt in blauen „Mundcontainern“, die im Mund versteckt und bei Bedarf verschluckt werden können.

Abb. 11, rechts Methadon bereits seit Tausenden von Jahren. Auch ihre teiloder vollsynthetischen Derivate, die Opioide der Neuzeit, bergen ein großes Missbrauchspotenzial.

- Opiate wirken an $\mu-$, k-, $\delta$-Opioidrezeptoren, die sich in der grauen Substanz des Gehirns sowie in der des Rückenmarks befinden.

- Der Körper selbst besitzt körpereigene Liganden wie das $\beta$-Endorphin, Metenkephalin und andere Substanzen [12].

- Die meisten Opiate und Opioide wirken analgetisch, sedierend, anxiolytisch, aber auch euphorisierend.

- Die somatischen Wirkeigenschaften der Opiate und Opioide sind Atemdepression, Übelkeit und Erbrechen, Bradykardie und Hypotonie, antitussive und gastrointestinale Wirkungen (Obstipationen) sowie Harnverhalt und Koliken (z. B. Urether, Galle).

Heroin Heroin ist ein Morphinderivat und kommt in unterschiedlichen Herstellungsstufen (Heroin I-IV) in den Verkehr: als braunes oder weißes Pulver bzw. als Granulat mit unterschiedlichen Strecksubstanzen ( $\bullet$ Abb. 9). Üblich ist ein Verschnitt mit 5-30\% Heroinanteil.

Heroin kann je nach Herstellungsstufe geraucht bzw. oral, intranasal oder intravenös appliziert werden ( $\odot$ Abb. 10). Der Heroinrausch dauert etwa 5 h. Mit Stimulanzien (Koffein, Kokain, Ephedrin), die dem Heroinverschnitt zugesetzt werden, kann eine Antriebssteigerung erreicht werden.

Substitutionspräparate Levomethadon (L-Polamidon $^{\circledR}$ ) und d,l-Methadon (Methadon ${ }^{\circledR}$; $\triangleright$ Abb. 11) werden zur Substitutionsbehandlung eingesetzt. Sie verursachen kein Rauscherleben und haben eine wesentlich längere Halbwertszeit.

- Opiathaltige Schmerz- und Hustenmittel (z.B. Pethidin, Kodein) werden ebenfalls häufig missbräuchlich zur Substitution oder in suizidaler Absicht, bei Kindern oder alten Menschen auch akzidentiell eingenommen. 


\section{Stadieneinteilung und Symptome bei Opiatentzug}

\begin{tabular}{|c|c|c|c|c|}
\hline \multirow[t]{2}{*}{ Stadium } & \multirow[t]{2}{*}{ Symptome } & \multicolumn{3}{|c|}{ Entzug von } \\
\hline & & Morphin & Heroin & Methadon \\
\hline 0 & $\begin{array}{l}\text { Verlangen nach Opiaten und } \\
\text { Angst }\end{array}$ & $6 \mathrm{~h}$ & $4 \mathrm{~h}$ & $12 \mathrm{~h}$ \\
\hline 1 & $\begin{array}{l}\text { Gähnen, Hyperhidrosis, Tränen- } \\
\text { fluss Rhinorrhoe, „Yen-Schlaf“ }\end{array}$ & $14 \mathrm{~h}$ & $8 \mathrm{~h}$ & $32-48 \mathrm{~h}$ \\
\hline 2 & $\begin{array}{l}\text { Zusätzlich zu Stadium 1: } \\
\text { Mydriasis, Piloerektion, Tremor, } \\
\text { Muskelschmerzen, Hitze- und } \\
\text { Kältegefühle, Knochen- und } \\
\text { Muskelschmerzen, Anorexie }\end{array}$ & $16 \mathrm{~h}$ & $12 \mathrm{~h}$ & $48-72 h$ \\
\hline 3 & $\begin{array}{l}\text { Zusätzlich zu Stadium 2: } \\
\text { Schlaflosigkeit, Blutdruck- und } \\
\text { Temperatursteigerung, Tachy- } \\
\text { kardie, Steigerung von Atemfre- } \\
\text { quenz und Atemtiefe, Übelkeit, } \\
\text { psychomotorische Unruhe }\end{array}$ & 24-36h & $18-24 h$ & $>49 \mathrm{~h}$ \\
\hline 4 & $\begin{array}{l}\text { Zusätzlich zu Stadium 3: } \\
\text { Fieber, Erbrechen, Diarrhoen, } \\
\text { Gewichtsverlust, Spontanejaku- } \\
\text { lation und Muskelkrämpfe, } \\
\text { Hämokonzentration mit } \\
\text { Leukozytose, Eosinopenie, } \\
\text { Anstieg von Blutzucker und } \\
\text { Laktat }\end{array}$ & $36-48 h$ & $24-36 h$ & \\
\hline
\end{tabular}

Tab. 4 nach [13]
Der langjährige Missbrauch von Opiaten und Opioiden führt zu Folgeerkrankungen.

- Psychisch: Lethargie, Apathie, Antriebsschwäche, dysphorische Stimmungslage sowie Depressionen, Angst- und Panikzustände, psychotische Episoden

- Physisch: Zahnschäden, Spritzenabszesse, Vaskulitiden, Embolien, Endokarditiden, Infektionskrankheiten [12].

Intoxikation Überdosierungen können bewusster oder akzidentieller Natur sein.

- Bei $40 \%$ der Intoxikationen handelt es sich um versehentliche Überdosierungen,

- $60 \%$ der Intoxikationen geschehen in suizidaler Absicht [4].

$\mathrm{Zu}$ den akzidentiellen Überdosierungen kommt es zumeist durch den i.v.-Konsum von Heroinverschnitt mit hohem Reinheitsgrad, aber auch durch den Konsum nach längerer Abstinenzphase (bei wieder erhöhter Opiatempfindlichkeit) und das versehentliche Öffnen verschluckter, heroingefüllter Beutel bei Kurieren (BodypackerSyndrom; sogenannte Mundcontainer zeigt - Abb. 10). Symptomatisch steht bei einer Opiatintoxikation die Ateminsuffizienz im Vordergrund ( Tab. 3). In der Folge können toxisches Lungenödem, Hirnödem, Rhabdomyolyse mit Nierenversagen und epileptische Krampfanfälle bzw. Status epilepticus und schwerste allergische Reaktionen durch Streck- und Bindemittel auftreten. Bei Mischintoxikationen oder präfinal ist die Miosis nicht obligat.
Versorgung Im Vordergrund steht auch hier die symptomatische Versorgung des Patienten.

- Bei schwerer Ateminsuffizienz sollte zum Aspirationsschutz frühzeitig intubiert und beatmet werden - wegen der Gefahr eines toxischen Lungenödems.

- Das toxische Lungenödem wird mittels PEEP-Beatmung, Diuretika (z.B. Furosemid $40 \mathrm{mg}$ i.v.) und Kortikosteroiden (z.B. Methylprednisolon $250 \mathrm{mg}$ i.v.) therapiert. Nur in schwersten Fällen sollte das Antidot Naloxon (z.B. Narcanti ${ }^{\circledR} 0,4 \mathrm{mg}$ in $0,9 \% \mathrm{NaCl} 1$ : 10 verdünnt, fraktioniert bis max. $2 \mathrm{mg}$ ) verabreicht werden.

- Das akut durch Naloxon ausgelöste Entzugssyndrom kann Erbrechen, Krampfanfällen, Asystolie, Lungenödem und Erregungszustände mit Aggressivität auslösen.

- Zudem ist die Halbwertszeit von Naloxon deutlich geringer als etwa die von Heroin, und es besteht die Gefahr, dass ein antagonisierter Patient erneut eintrübt.

- Buprenorphin (z.B. Subutex ${ }^{\circledR}$ ) ist durch Naloxon sehr schwer antagonisierbar.

Je nach Zustand des Patienten kann auf Intensivstationen Naloxon über Perfusor gegeben werden. In anderen Situationen müssen nach ausführlicher Abwägung ggf. auch größere Mengen direkt i.v. gegeben werden.

Entzug Der Opiatentzug ist für Süchtige höchst unangenehm.

- Vegetative Symptome wie Fieber, Schmerzen, Unruhe und gastrointestinale Krämpfe kennzeichnen die akute Entzugsphase.

- Je nach konsumierter Substanz und Halbwertszeit treten Symptome nach unterschiedlicher Abstinenzdauer auf ( Tab. 4).

Bei Morphin und Heroin beginnen 4-6 h nach der letzten Injektion milde Entzugserscheinungen, die sich ohne Opiatzufuhr in den folgenden Stunden steigern und ihren Höhepunkt nach 24-48 h erreichen.

- Trotz der oft beeindruckenden Symptomatik, die in der Entzugsphase auftritt, ist der Opiatentzug nicht lebensbedrohlich.

Versorgung Empathisches Vorgehen ist für den Notarzt oberstes Gebot. Die Therapie sollte symptomatisch erfolgen - Ziel sollte sein, den Patienten in eine stationäre, gestützte Entzugstherapie zu überführen. Drogenerfahrene Patienten versuchen nicht selten, den Notarzt zu manipulieren, z.B. um Opiate zu erhalten oder um eine stationäre Therapie abzuwenden. Zur Sedierung von agitierten Patienten sollten Benzodiazepine (z.B. Diazepam 5-10 mg i.v.) eingesetzt werden. 
Opiatintoxikationen enden mitunter letal. Absichtliche und akzidentielle Überdosierungen kommen nahezu gleich häufig vor. Infektionskrankheiten treten bei Opiatabhängigen gehäuft auf. Der Entzug ist eindrucksvoll, aber nicht vital bedrohlich. Antidota bleiben dem absoluten Notfall vorbehalten.

Fazit Drogenkonsum ist häufig in Deutschland, und die Zahl der Notfälle im Zusammenhang mit Drogen ist relevant. Drogenassoziierte Notfälle bergen durch ihre oftmals eindrücklichen Verläufe, das Umfeld, in dem sie vorkommen, oder die geringe Wertschätzung dem Süchtigen gegenüber das Risiko, dass wichtige Differenzialdiagnosen übersehen werden. Die oftmals unbekannte Zusammensetzung von Rauschmitteln im illegalen Verkauf sollte den Notarzt stets davon abhalten, sich in Sicherheit zu wiegen oder Notfälle im Zusammenhang mit Drogen zu bagatellisieren.

\section{Kernaussagen}

Notfälle im Zusammenhang mit illegalen Drogen sind oft eine Indikation für Notarzteinsätze.

Intoxikationen sind oftmals in suizidaler Absicht herbeigeführt.

Die Bewusstseinseinschränkung unter dem Einfluss von Drogen bedingt viele Notfallsituationen (psychischer Erregungszustand, Trauma, Entzugssymptomatik)

Die Behandlung der meisten Intoxikationen mit illegalen Drogen ist häufig auf die symptomatische Therapie und das Sichern der Vitalfunktionen beschränkt.

Antidota sollten zurückhaltend eingesetzt werden und dem absoluten Notfall vorbehalten bleiben.

Beim Vorliegen psychotischer Symptome sollten auch Antipsychotika zum Einsatz kommen.

Ein besonderes Augenmerk sollte auf die Abklärung möglicher Differenzialdiagnosen und zusätzlicher Diagnosen gelegt werden.

Patienten in Entzugssituationen sollten unterstützt und ermutigt werden: zur weitergehenden Abstinenz und zum Aufsuchen weiterführender Hilfen (z. B. Beratungsstellen).

Beim Vorliegen psychischer Symptome sollte die fachärztliche Abklärung erfolgen.

Der Eigenschutz hat in allen Situationen Vorrang.

\section{Hinweis}

Teil 2 dieses Beitrags erscheint in der nächsten AINS-Ausgabe.

\section{Literaturverzeichnis}

1. Drogenbeauftragte der Bundesregierung. Drogen und Suchtbericht 2008. Berlin: Bundesministerium für Gesundheit; 2008

2. Vitale S, van de Mheen D. Illicit drug use and injuries: A review of emergency room studies. Drug Alcohol Depend 2006; 82(1): 1-9

3. Pajonk FG, Bartels HH, Grünberg KAS, Moecke Hp. Psychiatrische Notfälle - Häufigkeit und Versorgung im Vergleich einer großstädtischen mit einer ländlichen Region. Notfall Rettungsmed 2002; 5: 110-115

4. Pfab R, Eyer F, et al. Cause and motivation in cases of non-fatal drug overdoses in opiate addicts. Clin Toxicol (Phila) 2006; 44(3): 255-259

5. Bonnet U. Behandlung der Cannabisabhängigkeit bei Erwachsenen. Psychoneuro 2006; 32 (11): 541-546

6. Schlimme J, Rada D, Schneider U. Cannabiskonsum und seine psychosozialen Wirkungen im Kulturkreis. Fortschritte Neurologie Psychiatrie 2001; 69: 367-373

7. Schneider U, Seifert J. Neurobiologie des Cannabinoidsystems. Psychoneuro 2006; 32 (11): 532-535

8. Soyka M. Drogen- und Medikamentenabhängigkeit. In: Möller HJ, Laux G, Kapfhammer HP.(Hrsg.) Psychiatrie und Psychotherapie. Band 2: Spezielle Psychiatrie. 3. Aufl. Heidelberg: Springer; 2008: 187-241

9. Ebert D. Psychiatrie systematisch. Bremen: Uni-Med; 2003

10. Preuss UW, Bahlmann M, Koller G, Soyka M. Die Behandlung der Kokainabhängigkeit. Intoxikation, Entzug und Rückfallprophylaxe. Fortschritte Neurologie Psychiatrie 2000; 68: 224-238

11. Garwin FH, Kleber HD. Abstinence symptomatology and psychiatric diagnoses in cocain abusers. Arch Gen Psychiatry 1986; 43: 107-113

12. Gölz J. Der drogenabhängige Patient. Handbuch der schadensmindernden Strategien. München - Jena: Urban \& Fischer; 1999

13. Kardels B, Kinn M, Pajonk FGB. Akute psychiatrische Notfälle. Stuttgart: Thieme; 2007

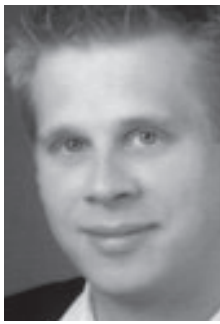

Michael Kinn ist Arzt in Weiterbildung der Abteilung für Anästhesie, Intensivmedizin und Schmerztherapie an der Berufsgenossenschaftlichen Unfallklinik Ludwigshafen.

E-Mail:

mkinn@bgu-ludwigshafen.de

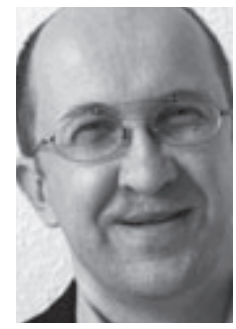

Dr. med. Rüdiger Holzbach leitet die Abteilung Suchtmedizin der Westfälischen Kliniken Warstein und Lippstadt. Sein wissenschaftlicher Schwerpunkt liegt auf dem Thema der Medikamentenabhängigkeit.

E-Mail: R.Holzbach@wkp-Iwl.org

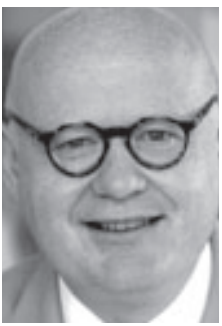

Prof. Dr. med. Frank G. B. Pajonk ist Chefarzt der Privat-NervenKlinik Dr. Kurt Fontheim in Liebenburg. Zu seinen wissenschaftlichen Schwerpunkten zählen Wirkungen von Psychopharmaka und psychiatrische Notfälle.

E-Mail:

pajonk@klinik-dr-fontheim.de

\section{Literatur online}

Das vollständige Literaturverzeichnis zu diesem Beitrag finden Sie im Internet:

Abonnenten und Nicht-

Abonnenten können unter „www.thiemeconnect.de/ejournals" die Seite der AINS aufrufen und beim jeweiligen Artikel auf „Ergänzendes Material" klicken - hier ist die Literatur für alle frei zugänglich. Abonnenten können alternativ über ihren persönlichen Zugang an das Literaturverzeichnis gelangen. Wie das funktioniert, lesen Sie unter: http://www.thiemeconnect.de/ejournals/ help\#SoRegistrieren 